\title{
Patrones de liderazgo en la cultura regional: un análisis intersectorial
} Leadership Patterns in the Regional Culture: An Inter-Sectorial Analysis

Patrões de liderança na cultura regional: uma análise intersetorial

\author{
Juan Carlos Espinosa \\ Francoise Contreras ${ }^{\star *}$ \\ Ana Fernanda Uribe \\ David Barbosa ${ }^{* * *+}$
}

Fecha de recibido: 22 de mayo de 2013

Fecha de aprobado: 10 de mayo de 2014

Doi: dx.doi.org/10.12804/rev.univ.empresa.27.2014.01

Para citar este artículo: Espinosa, J. C., Contreras, F., Fernanda, U. A., \& Barbosa, D. (2014). Patrones de liderazgo en la cultura regional: un análisis intersectorial. Universidad \& Empresa, 16(27), 45-61. doi: dx.doi.org/ 10. 12804/ rev.univ.empresa.27.2014.01

* Estudiante del doctorado en Ciencias de la Dirección de la Universidad del Rosario (Colombia). Magíster en Psicología de la Universidad Nacional de Colombia. Profesor de la Escuela de Administración de la Universidad del Rosario (Colombia). Correo electrónico: juanc.espinosa@urosario.edu.co

** Doctora en Psicología de la Universidad Autónoma de Madrid (España). Magíster en Evaluación de la Educación de la Universidad Santo Tomás (Colombia). Profesora Titular de la Escuela de Administración de la Universidad del Rosario (Colombia). Correo electrónico: francoise.contreras@urosario.edu.co

**** Doctora en Psicología Clínica y de la Salud de la Universidad de Granada, España). Magíster en Educación y Prevención del SIDA de la Universidad Complutense de Madrid (España). Profesora e investigadora líder del Grupo de investigación Productividad y Competitividad de la Universidad Pontificia Bolivariana, Bucaramanga (Colombia). Correo electrónico: anafernanda.uribe@upb.edu.co

***** Estudiante del doctorado en Ciencias de la Dirección de la Universidad del Rosario (Colombia). Magíster en Administración de la Universidad de los Andes (Colombia). Correo electrónico: david.barbosa@urosario.edu.co 


\section{RESUMEN}

El objetivo de este estudio es observar si existe un patrón característico de liderazgo en una cultura regional específica y analizar si se presentan diferencias significativas en razón del sector económico al que pertenecen las organizaciones (industria y servicio). Se trabajó con una muestra de 449 empleados de ocho empresas que operan en la ciudad de Bucaramanga. Se utilizó el Test de adjetivos de Pitcher - PAT — . De acuerdo con los resultados, sí existe un patrón de liderazgo caracterizado por los adjetivos: responsable, humano, trabajador, colaborador, honesto y amable. En general, las características de liderazgo en los dos sectores estudiados fueron muy similares, aunque se observaron algunas diferencias. En este texto se discuten estos hallazgos.

Palabras clave: estilos de liderazgo, liderazgo y cultura, patrones de liderazgo.

\section{ABSTRACT}

The purpose of this study is to verify whether or not there is a characteristic pattern of leadership in a specific regional culture and also whether there are any significant differences according to the economic sector to which the organization belongs (industry and services). A sample of 449 employees of eight companies that operate in the city of Bucaramanga was used. The Pitcher Adjectives Test (PAT) was applied. According to the results, a leadership pattern characterized by the following adjectives: responsible, human, hardworking, collaborative, honest and pleasant, does exist. In general, the characteristics of leadership in the two sectors studied were very similar, although certain differences were noted. In this text the findings are discussed.

Keywords: Leadership and culture, leadership patterns, leadership styles.

\section{RESUMO}

O objetivo deste estudo é observar se existe um patrão característico de liderança em uma cultura regional específica e analisar se apresentam-se diferenças significativas por causa do setor económico ao que pertencem as organizações (indústria e serviço). Trabalhou-se com uma amostra de 449 empregados de oito empresas que operam na cidade de Bucaramanga. Utilizou-se o Teste de adjetivos de Pitcher - PAT - . De acordo com os resultados, existe sim um patrão de liderança caracterizado pelos adjetivos: responsável, humano, trabalhador, colaborador, honesto e amável. Em geral, as características de liderança nos dois setores estudados foram muito similares, ainda que se observaram algumas diferenças. Neste texto discutem-se estes resultados.

Palavras-chave: estilos de liderança, liderança e cultura, patrões de liderança. 


\section{INTRODUCCIÓN Y REVISIÓN DE LA LITERATURA}

Tradicionalmente los aspectos culturales en relación con el liderazgo no han recibido la suficiente atención por parte de los investigadores. Actualmente se observa en los estudios de este tema una clara tendencia a considerar los aspectos contextuales y culturales en el ejercicio del liderazgo (Castro \& Nader, 2004). Una de las investigaciones más importantes al respecto es la de Globe. Esta permitió encontrar que la cultura empresarial y los atributos del líder están estrechamente relacionados y que la cultura nacional y la cultura organizacional influyen en el ejercicio del liderazgo. Estos resultados señalan entonces la necesidad de comprender los aspectos culturales que se relacionan con el liderazgo y resaltan la importancia de aplicar estos conocimientos a la formación de líderes con el fin de potenciar su capacidad para desempeñar su rol en medio de la complejidad propia de los mercados actuales, los cuales son cada vez más globales y menos locales (Javidan, Dorfman, Howell, \& Hanges, 2010).

La cultura puede entenderse como "la programación colectiva de la mente que permite diferenciar a los miembros de un grupo o categoría de personas, de otros" (Hofstede, 1991, p. 5). Esta definición puede aplicarse no solo al ámbito nacional sino también a organizaciones y grupos empresariales. Respecto a la gestión, en particular, se encuentra el concepto de la relatividad cultural. Este concepto determina que los ambientes humanos en los cuales opera una organización pueden afectar los procesos de dirección (Hofstede, 1994). En este sentido, la cultura de un país o región afecta ineludiblemente a los líderes, así como la forma en la que estos dirigen las organizaciones y como las prácticas directivas son percibidas por los colaboradores (Hofstede, 1994; Kogut \& Singh, 1988; Molero, 2002; Semenov, 2000).

Hofstede, Van Deusen, Mueller y Charles (2002) analizaron en quince países las metas de negocio perseguidas por los líderes, en el marco de los procesos de globalización. Los resultados de su estudio les permitió, por un lado, comprender que las culturas nacionales - aquellas que son compartidas por los habitantes de un país - están conformadas por una serie de subculturas que difieren entre sí en virtud de aspectos tales como los grupos sociales, la religión y la composición étnica, entre otros (Molero, 2002), y, por el otro, jerarquizar a estos países en siete grupos, a partir de los cuales 
identificaron varias categorías a las que llamaron arquetipos, en virtud de sus prácticas de liderazgo. Un hallazgo importante de este estudio fue el verificar que tanto la percepción como la cultura nacional correlacionaron significativamente con el nivel de riqueza del país.

Castro y Lupano (2005), por su parte, encontraron diferencias significativas entre los individuos respecto a la percepción de las características que le atribuyen a un líder considerado como efectivo. La categoría definida previamente como 'buenos líderes' correspondía a aquellas personas carismáticas y orientadas al logro, lo cual se dio más en culturas dinámicas y flexibles, comprometidas institucionalmente y con metas a largo plazo. En el estudio de estos autores se concluye que, efectivamente, dependiendo de la cultura, predominan distintos tipos de líder.

Contreras, Barbosa, Juárez, Uribe y Mejía (2009) observaron en cuatro ciudades de Colombia (Barranquilla, Bogotá, Bucaramanga y Cali) los estilos de liderazgo en relación con otras variables como la presencia de riesgos psicosociales y el clima organizacional en entidades promotoras de salud -EPS- A pesar de tratarse del mismo país, los autores encontraron algunas diferencias significativas entre ciuda- des. Esto puede obedecer a las subculturas propias del país, tal como lo señaló Hofstede (1994).

De acuerdo con el citado estudio, aunque se observó alta presencia de riesgos psicosociales en las cuatro ciudades, Bucaramanga presentó los niveles más altos, especialmente en el factor de relaciones interpersonales, el cual fue mucho más relevante en esta ciudad. Cabe recordar que las características principales de este factor de riesgo están relacionadas con la comunicación, la resolución de conflictos entre el grupo, valores éticos, compromiso, confianza, respeto y tolerancia, entre otros, todo ello unido a un bajo apoyo percibido del líder al compararse con las otras ciudades estudiadas (Contreras et al., 2009). Un aspecto que reviste especial importancia es que en esta investigación el estilo de liderazgo considerado como no deseable se relacionó con un mayor bienestar del trabajador, en otras palabras, muchas características que en la literatura se consideran como no deseables en el liderazgo correlacionaron positivamente con un menor efecto de los riesgos psicosociales. Esto guardó relación con la percepción de un clima organizacional caracterizado principalmente por altos niveles de control (Contreras, Barbosa, Juárez, \& Uribe, 2010). Estos hallazgos 
denotaron un posible efecto cultural que motivó el interés de seguir estudiando este fenómeno con un mayor detenimiento.

Se consideró importante incluir, además del sector industrial, el de servicios. Esto debido no solo a la necesidad de observar su relación con el liderazgo sino también a considerar su importancia para los países tanto desarrollados como en vías de desarrollo, países en los que la participación del sector terciario tanto para el empleo como en los ingresos bordea el $70 \%$ (Barreiro, de Andrade, \& Salgueiro, 2012). Lo anterior se debe al importante incremento de este sector en los últimos años, algo que se ha hecho más evidente desde comienzos de este siglo, un momento en el que las economías industrializadas, tal como lo indican Maroto-Sánchez, (2010), se convirtieron en economías de servicios.

En relación con lo anterior, Martínez y Lázaro (2007) hablan del surgimiento de la denominada 'ciencia de los servicios y de las habilidades', que deberán desarrollar los directivos de las empresas de servicios, pues estas involucran una serie de competencias complementarias a las que el directivo del tradicional mercado industrial poseía. Destacan, básicamente, saberes de tipo tecno- lógico, conocimiento del negocio y socio-organizativos que involucran capacidades para coordinar, motivar y gobernar equipos de trabajo con especial énfasis en aspectos multiculturales.

En este contexto investigativo, el propósito de este estudio es caracterizar el estilo de liderazgo percibido por los trabajadores en el área metropolitana de Bucaramanga, esta vez con una población distinta a la del sector salud. En esta ocasión se trabaja con dos sectores diferentes - servicios e industrial - para considerar las diferencias que pueden obedecer al tipo de organización y quizás no directamente a la cultura, pues es probable que el estilo de liderazgo percibido esté en función del sector al que pertenece la empresa.

Debido a lo anterior, los análisis se realizan de forma global por sectores, con el fin de minimizar el posible efecto de las subculturas que suelen darse en determinados tipos de organizaciones. Se hace mención al sector primario o agropecuario, el cual obtiene sus productos de la naturaleza de manera directa, sin ningún proceso de transformación; el sector secundario o industrial, en el cual se dan las transformaciones de los productos, y el sector terciario o de servicios, el cual, a pesar de noproducirmercancías, esnecesario 
para el funcionamiento de la economía (Banco de la República, 2014).

\section{METODOLOGÍA}

\section{A. Diseño}

Descriptivo-trasversal, de comparación entre grupos.

\section{B. Participantes}

Se trabajó con una muestra no probabilística de 449 trabajadores, pertenecientes a ocho empresas del área metropolitana de Bucaramanga. El $35,6 \%$ (160) trabajaban en el sector industrial y el 64,4\% (289) en el de servicios. Más de la mitad de los participantes fueron hombres $(66,4 \%$, $)$, con una edad promedio de 32,8 años $\left(\mathrm{SD}^{1}=9,38\right)$. El tiempo de vinculación a la organización fue de 4,89 años en promedio $(\mathrm{SD}=5,99)$.

\section{Instrumento}

En este estudio se utilizó el Test deadjetivos de Pitcher - PAT - (1997a). Este instrumento está compuesto por sesenta adjetivos a través de los cuales los subordinados evalúan su jefe. Aunque evalúa tres estilos de liderazgo, que la autora denomina artesanos, tecnócratas y artistas (Pitcher, 1997b, 1999), en esta investigación se analizan los adjetivos de forma independiente, sin categorías previas y solo teniendo en cuenta la frecuencia de uso. Juárez, Contreras y Barbosa (2013) encontraron que las propiedades psicométricas del test son adecuadas al analizar una muestra de 400 trabajadores del sector salud en Colombia.

\section{Procedimiento}

Previa autorización de las directivas de las organizaciones y la obtención del consentimiento informado de los participantes se inició la aplicación del instrumento de forma colectiva (por grupos de aproximadamente diez personas). Se pidió a los empleados evaluar el estilo de liderazgo de sus jefes/superiores a partir del test de Pitcher. La participación fue voluntaria y anónima. Los trabajadores que hicieron parte del estudio no recibieron ningún tipo de retribución.

\section{RESULTADOS}

A nivel sociodemográfico la muestra estuvo compuesta por mujeres y hombres, siendo mayor este último grupo $(66,4 \%)$. Más de la mitad de los participantes pertenecen al sector de servicios $(64,4 \%)$ y tienen una contratación a término indefinido

\footnotetext{
SD: Standard Deviation (desviación estándar).
} 
en un alto porcentaje (cercano al $70 \%$ ). La interacción de estos tres aspectos contó con un mayor número de participantes. En contraste, el menor número de participantes se congregó en el grupo de mujeres del sector industrial vinculadas a la organización por medio de contratos de prestación de servicios (figura 1). Se encontró similitud en las jornadas laborales por turnos y el horario de oficina ( $52 \%)$, la mayoría reporta tener pareja $(57,2 \%)$ y ser padres $(61,5 \%)$ siendo lo más frecuente que tengan 1 o 2 hijos (con 27,2\% y $22,7 \%$, respectivamente).

Figura 1. Distribución de participantes por sector, sexo y tipo de contratación

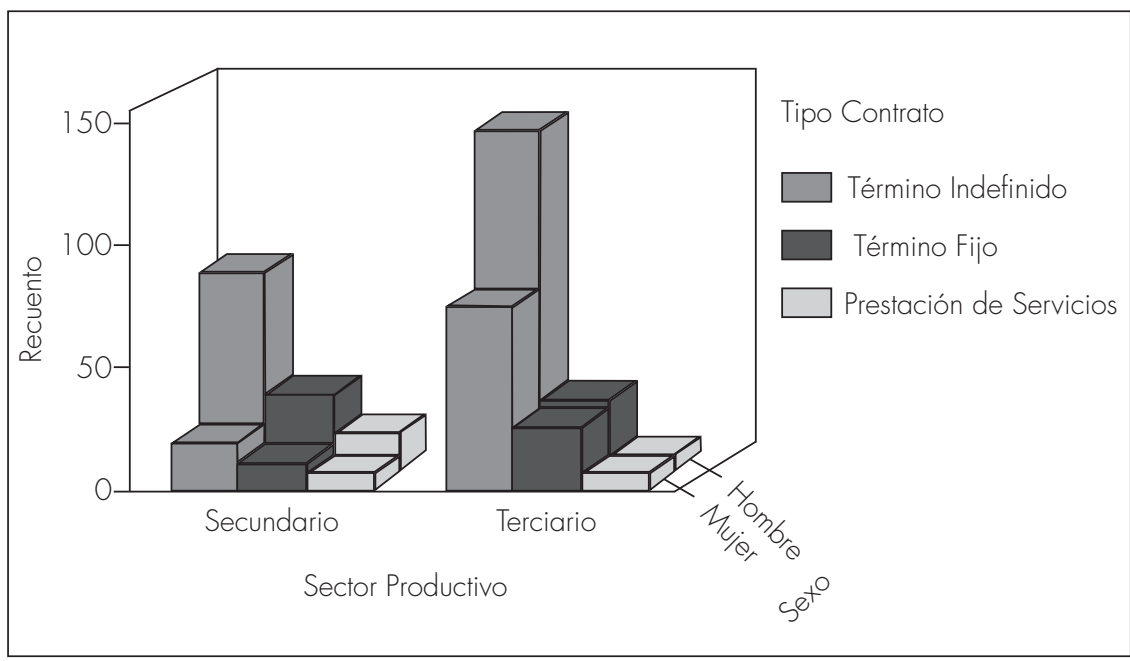

Fuente: elaboración propia.

En el test de Pitcher los trabajadores seleccionaron entre sesenta adjetivos aquellos que mejor describen a su jefe. El porcentaje de elección de estos adjetivos osciló entre 5,15\% y $69,35 \%$, siendo el promedio 27,15 y la desviación típica 15,76. Los adjetivos se agruparon en tres categorías: poco característicos, característicos y más característicos. Esta agrupación se realizó con base en el punto de corte $\mathrm{M} \pm 0,5 \mathrm{SD}$, es decir, $27,15 \pm 7,88$.

De acuerdo con esta agrupación los adjetivos poco característicos fueron elegidos por el $\leq 19 \%$ de los trabajadores. En el segundo grupo (característicos) se encontraron los adjetivos que tuvieron porcentajes de elección entre el $20 \%$ y $34 \%$. En el grupo final (más característicos) 
se ubicaron los adjetivos que presentaron $\geq 35 \%$ de preferencia para describir el liderazgo del jefe.

Posteriormente, se identificó en cada adjetivo si existía o no una diferencia estadísticamente significativa entre los porcentajes de elección de los trabajadores de los sectores secundario y terciario. Esta estimación se realizó con base en una prueba de diferencia de proporciones, por lo que fue necesario expresar en proporciones $(0$ a 1$)$ los valores porcentuales ( 0 a 100). Se aceptó como significativa la diferencia que presentara un $\mathrm{p}<0,05$. A continuación se analiza el porcentaje de elección de cada uno de los adjetivos estu- diados y se indican aquellos en los cuales los sectores se diferencian de manera significativa.
A. Adjetivos más característicos para describir el liderazgo en Bucaramanga

En este primer grupo se ubican dieciséis de los sesenta adjetivos del PAT que fueron seleccionados $\geq 35 \%$. El adjetivo más usado para referirse al liderazgo en Bucaramanga fue 'responsable', el cual fue seleccionado por el $69 \%$ de los trabajadores para describir a su jefe. Esta elección se realizó sin importar el sector productivo de los participantes (figura 2).

Figura 2. Adjetivos del PAT más característicos para describir a los líderes en Bucaramanga

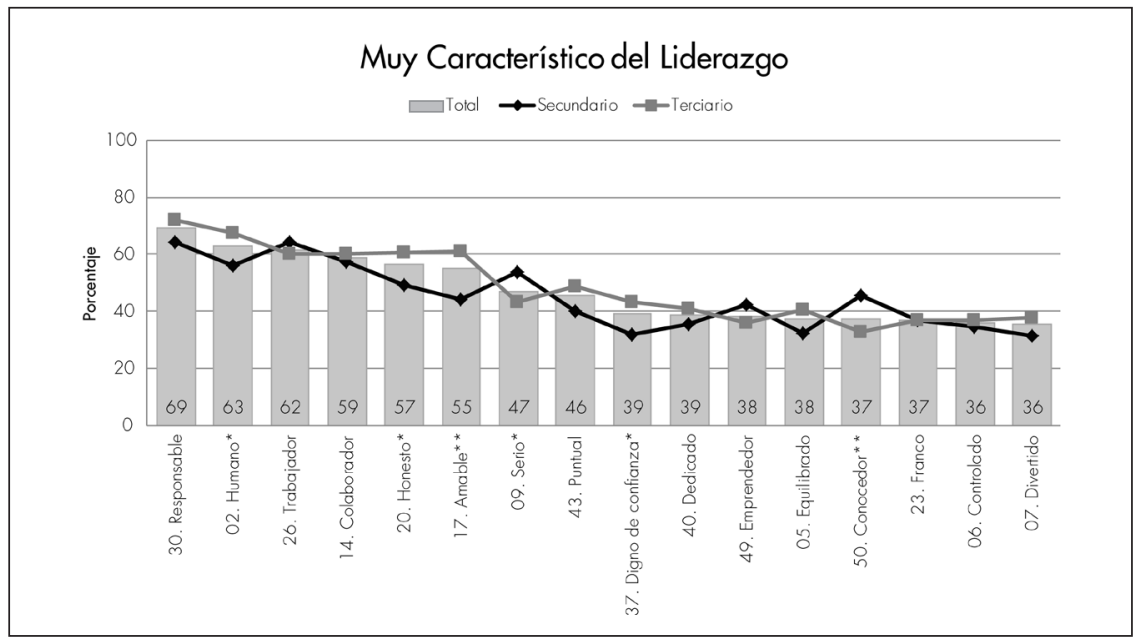

$* * \mathrm{p} \leq, 01 ; * \mathrm{p} \leq, 05$ representan diferencias intersectoriales estadísticamente significativas

Fuente: elaboración propia. 
Estos dieciséis adjetivos que conforman el grupo de aspectos más característicos de los líderes bumangueses se pueden reunir en cuatro subgrupos con el fin de realizar un análisis más detallado. El primer subgrupo está conformado por los adjetivos que fueron seleccionados por más del $60 \%$ de los trabajadores para describir a sus jefes. En este primer subgrupo se ubican los términos responsable (69\%), humano $(63 \%)$ y trabajador $(62 \%)$; sin embargo, el calificativo humano es significativamente más elegido por los trabajadores del sector terciario que por los del sector secundario, es decir, para los trabajadores este adjetivo es de los más característicos de los lideres bumangueses pero lo es aún más en los líderes del sector terciario.

En un segundo subgrupo se ubican los adjetivos colaborador, honesto y amable con $59 \%, 57 \%$ y $55 \%$ de elección, respectivamente. En este subgrupo honestidad y amabilidad son reconocidas como algo más característico en sus líderes en mayor medida por los trabajadores del sector terciario que por los trabajadores del sector secundario.

Un tercer subgrupo de los adjetivos más característicos para describir el liderazgo en Bucaramanga lo conforman serio y puntual, con porcen- tajes globales muy similares $(47 \%$ y $46 \%$, en su orden). La importancia de estos calificativos está intercambiada para los trabajadores del sector secundario y terciario. Ser serio es más importante en el sector secundario y ser puntual es más relevante en el sector terciario, no obstante, solo en el adjetivo serio la diferencia fue estadísticamente significativa.

El cuarto subgrupo estuvo conformado por los adjetivos digno de confianza (39\%), emprendedor (38\%), equilibrado(38\%), conocedor(37\%), franco $(37 \%)$, controlado (36\%) y divertido (36\%). Digno de confianza y conocedor presentaron diferencias significativas en la apreciación de los subalternos de los sectores secundario y terciario: para los primeros es más relevante ser conocedor, mientras que para los segundos lo es ser digno de confianza.

\section{B. Adjetivos característicos para describir el liderazgo en Bucaramanga}

En este grupo se ubican los adjetivos cuyo porcentaje de elección para describir a los jefes por parte de 449 subalternos de los sectores secundario y terciario osciló entre $20 \%$ y $34 \%$. El grupo está conformado por veintiuno de los sesenta adjetivos de la lista propuesta por 
Pitcher (1997a). Con fines analíticos, este grupo se divide en tres subgrupos: a) $34 \%$ a $30 \%, b) 29 \%$ a $25 \%$ y c) $24 \%$ a $20 \%$ (figura 3 ).

En el primer subgrupo (34\% a 30\%) se ubican los adjetivos razonable, orientado a personas, enérgico, estable, generoso, firme, abierto, cortés y fácil de llevar. En este subgrupo se aprecia que en todos los casos los adjetivos son más utilizados por los trabajadores del sector terciario que por los del sector secundario; sin embargo, solo en el término cortés se presentan diferencias significativas en los porcentajes presentes en ambos sectores, siendo este significativamente más usado para describir a los líderes del sector terciario.

Figura 3. Adjetivos del PAT característicos para describir a los líderes en Bucaramanga

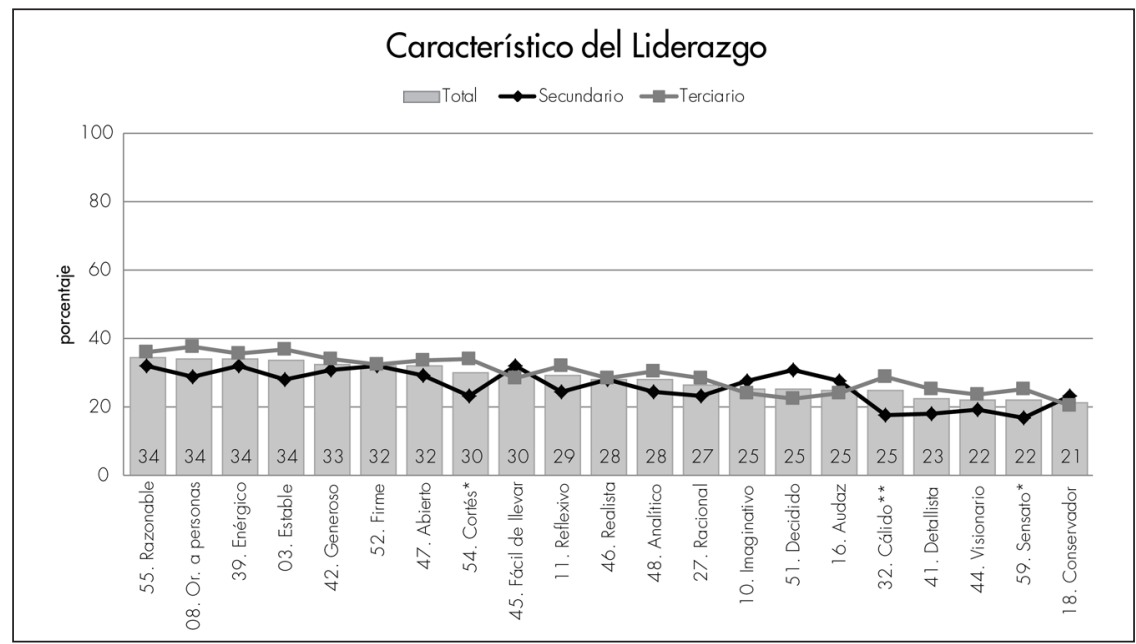

$* * \mathrm{p} \leq, 01 ; * \mathrm{p} \leq, 05$ representan diferencias intersectoriales estadísticamente significativas

Fuente: elaboración propia.

En el segundo subgrupo (29\% a $25 \%)$ se encuentran los adjetivos reflexivo, realista, analítico, racional, imaginativo, decidi $i d o$, audaz y cálido. El análisis por sectores refleja que reflexivo es lo más característico de este subgrupo para el sector terciario, mientras que para el sector secundario lo es ser decido; no obstante, solo en el calificativo cálido se aprecian diferencias estadísticamente significativas donde los trabajadores del sector secundario reportan un porcentaje inferior al reportado por el sector terciario. 
El tercer subgrupo (24\% a $20 \%)$ recoge los adjetivos detallista, visionario, sensato y conservador. En este subgrupo se aprecian diferencias estadísticamente significativas entre los líderes del sector secundario y terciario en ser sensato, lo cual es reportado en mayor porcentaje por los empleados del sector terciario.

\section{Adjetivos poco característicos para describir el liderazgo en Bucaramanga}

En este último grupo se ubican los adjetivos que fueron usados por menos del 20\% de los trabajadores bumangueses que participaron en el estudio para describir a sus líderes. El grupo está conformado por vein- titrés de los sesenta adjetivos del listado de Pitcher (1997a). Con fines analíticos, se divide el grupo en 3 subgrupos: a) $15 \%$ a $19 \%, b) 14 \%$ a $10 \%$ y $c$ ) menos del $10 \%$ (figura 4 ).

En el primer subgrupo (15\%a 19\%) se encuentran los adjetivos sabio, impredecible, rígido, intuitivo, meticuloso, brillante, emocional y cerebral. De este subgrupo solo se identificaron diferencias estadísticas en el adjetivo rígido, el cual es significativamente más usado por los trabajadores del sector secundario que por los del sector terciario. De hecho, para el sector secundario, este término puede ubicarse como característico mientras que para el sector terciario haría parte de los adjetivos poco característicos.

Figura 4. Adjetivos del PAT poco característicos para describir a los líderes en Bucaramanga

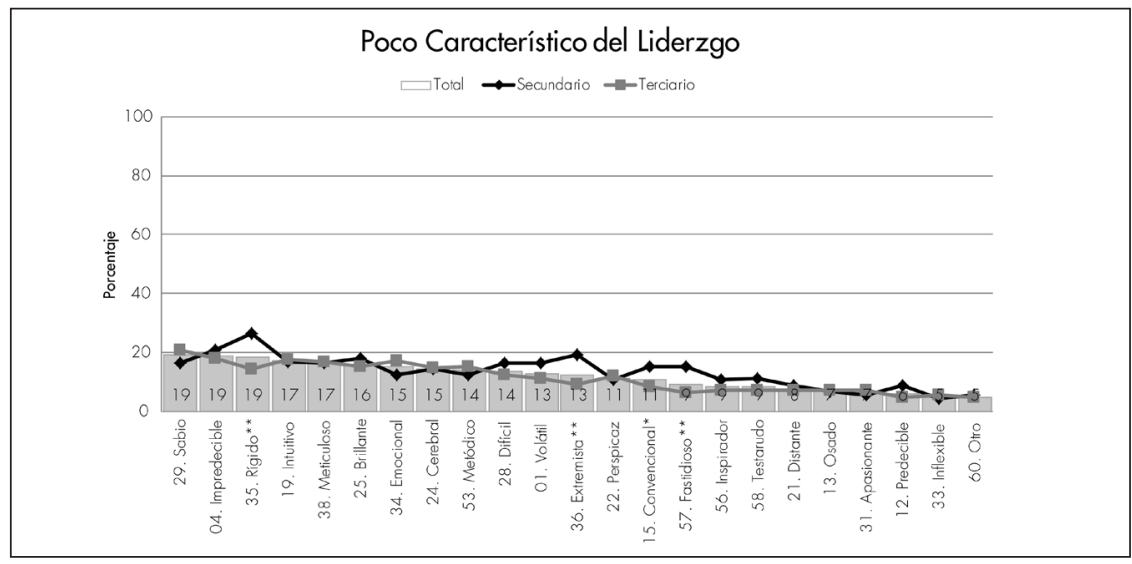

${ }^{* *} \mathrm{p} \leq, 01 ; * \mathrm{p} \leq, 05$ representan diferencias intersectoriales estadísticamente significativas

Fuente: elaboración propia 
El segundo subgrupo (10\% a 14\%) está conformado por los adjetivos metódico, difícil,volátil, extremista, perspicaz y convencional. Los análisis efectuados permiten concluir que los calificativos extremista y convencional son usados significativamente más por parte de los trabajadores del sector secundario que por los del sector terciario.

Finalmente, en el tercer subgrupo se ubican los adjetivos que fueron elegidos por menos del 10\% de los 449 subalternos para describir a sus líderes. Este grupo está conformado por los términos: fastidioso, inspirador, testarudo, distante, osado, apasionante, predecible e inflexible. Exceptuando el calificativo fastidioso, que es más usado por parte de los trabajadores del sector secundario, todos los adjetivos son usados de la misma manera por los trabajadores bumangueses.

\section{DISCUSIÓN Y \\ CONCLUSIONES}

El liderazgo en Bucaramanga, según el presente estudio, conserva un patrón específico caracterizado principalmente por dieciséis adjetivos (figura 2). De estos, seis presentan diferencias estadísticamente significativas entre los sectores secundario y terciario. Esto denota el posible efecto de la cultura regional asociada al liderazgo, un aspecto que ha sido evidenciado en estudios previos (Hofstede et al., 2002; Molero, 2002).

Se confirma entonces el impacto que tiene la cultura regional en los líderes y la forma como estos son percibidos (Kogut \& Singh, 1988; Molero, 2002; Semenov, 2000). Las diferencias encontradas entre los dos sectores no fueron determinantes de un estilo distinto de liderazgo. Esto puede deberse a que como plantea Gallouj (2002, citado en Barreiro de Souza et al., 2012), los límites intra e intersectoriales son cada vez más tenues. Se está dando entonces un proceso de convergencia entre los sectores de tal suerte que el manufacturero se orienta cada vez más a los servicios y el de los servicios gradualmente se industrializa.

No obstante, es relevante analizar las diferencias encontradas entre los dos grupos, que se dan básicamente en los adjetivos humano, honesto, amable, serio, digno de confianza y conocedor. Estos calificativos, aunque son muy importantes para describir a los líderes bumangueses, es decir, son usados por los trabajadores de los dos sectores estudiados para describir a sus jefes, presentan diferencias en la frecuencia de uso. Se encontró que los términos humano, honesto, amable y digno de 
confianza se usan con mayor frecuencia en el sector terciario, mientras que serio y conocedor lo son más en el sector secundario. Este hallazgo parece estar relacionado con el privilegio, en el sector industrial, del control como mecanismo que favorece la eficiencia en los procesos y, por lo tanto, el trabajador percibe al jefe como alguien serio cuyas órdenes se cumplen de manera apropiada. Además, es propio del sector industrial que el poder experto, aquel que surge de los años de experiencia y la formación, sea una condición para ser directivo. De hecho, en los manuales de cargo los años de experiencia son un requisito indispensable para acceder a los puestos superiores. Esto explicaría que los directivos sean percibidos como personas que conocen su oficio.

En el sector terciario, como lo precisan Martínez y Lázaro (2007), los directivos tienen como uno de sus saberes centrales coordinar, motivar y gobernar equipos de trabajo. Afirman que "en términos muy básicos, lo que se está pretendiendo es conseguir un perfil mixto entre el tradicional ingeniero y el tradicional economista al que se le agregan componentes adicionales de habilidades interpersonales" (p. 125). Los adjetivos humano, honesto, amable y digno de confianza, corresponden a esta categoría de habilidades interpersonales. Las diferencias encontradas parecen obedecer al objetivo mismo de las organizaciones en uno y otro sector, esto es, mientras una compañía del sector industrial tiene un compromiso central con el uso eficiente de los recursos, lo cual exige esquemas de control rígidos, estructuras marcadamente jerarquizadas y procesos poco flexibles, una empresa de servicios requiere ser eficiente pero debe enfocarse en la eficacia, es decir, en la satisfacción adecuada de las necesidades del cliente.

Al parecer estamos ante el tránsito gradual de un modelo mental tradicional, en el que el directivo ve a la organización como una máquina, a verla desde un modelo más integral, desde una perspectiva sistémica. Esto aunque fue más evidente en las empresas de servicios es aplicable perfectamente a cualquier sector económico en la actualidad (Contreras \& Barbosa, 2013). Es probable que este hecho este relacionado con la tendencia general de privilegiar el estilo de liderazgo transformacional sobre el transaccional (Bass, 1999). Esto corresponde con el incremento del sector de servicios en la última década, en donde las economías industrializadas se convirtieron en economías de servicios (MarotoSánchez, 2010). 
En cuanto a las otras dos categorías de análisis, adjetivos característicos y poco característicos para describir el liderazgo en Bucaramanga (figuras 3 y 4), se encontró que tres de los veintiún adjetivos característicos presentan diferencias intersectoriales significativas, estos son cortés, cálido y sensato. Estos son más usados por los trabajadores del sector terciario para describir a sus líderes. La última agrupación la conformaron veintitrés adjetivos del PAT, los cuales aunque son poco usados para describir a los jefes por parte de los subalternos también involucran diferencias sectoriales en los calificativos rígido, extremista, convencional y fastidioso, siendo su uso un poco más frecuente en el sector secundario que en el terciario, aunque en ambos la frecuencia de uso es menor al $10 \%$.

Las reflexiones hechas en el apartado anterior, con base en lo expuesto por Martínez y Lázaro (2007) y Contreras y Barbosa (2013), permiten comprender estos resultados. En las empresas del sector terciario se espera que el estilo del directivo sea más transformacional con lo cual los adjetivos cortés, cálido y sensato son congruentes. Los términos rígido, extremista, convencional y fastidioso serían más propios de un estilo de liderazgo autocrático, el cual tiene algún grado de identidad con el liderazgo transaccional (Bass, 1999).
De los trece adjetivos (22\%) del PAT que presentan diferencias intersectoriales en la descripción del liderazgo por parte de 449 trabajadores, seis se ubican en la categoría de más característico, tres en el grupo de adjetivos característicos y cuatro en el grupo de adjetivos poco característicos. Este hallazgo confirma lo planteado por Hofstede et al. (2002) respecto a las diferencias culturales al interior de un mismo país y región. Estas diferencias intersectoriales son estadísticamente significativas en el $22 \%$ de los calificativos del test de adjetivos de Pitcher. Estos se encuentran presentes tanto en lo más característico como en lo menos característico del liderazgo en los directivos bumangueses, según la apreciación de 449 subalternos.

Las principales diferencias en los adjetivos de mayor uso en los dos sectores se presentaron en los términos humano, honesto y amable. Las diferencias intersectoriales en los adjetivos de poco uso se presentaron en los términos fastidioso, convencional y extremista. Este aspecto muestra que a pesar de pertenecer a una misma categoría de importancia, el sector del que hace parte la empresa muestra particularidades que deben atenderse con el objetivo de comprender mejor el liderazgo, es decir, se confirman los factores con- 
textuales presentes en el estudio del liderazgo (Bass \& Avolio, 1993).

En síntesis, la percepción de los subalternos para describir el liderazgo obedeció a la interacción de tres elementos previamente expuestos: 1) las subculturas que se estructuran en los países comparten en mayor o menor medida creencias y valores que las hacen similares en algún grado (Hofstede et al., 2002), 2) la transformación en la composición de los sectores económicos tradicionales que hacen cada vez más tenues sus límites debido a que en la actualidad las empresas industriales tienden a parecerse a las de servicios y viceversa (Barreiro de Souza et al., 2012) y 3) la tendencia de la transición del liderazgo transaccional al transformacional en ambos sectores. Esto último es más evidente en el sector de servicios. Esta transición se observa en que los adjetivos usados para describir el liderazgo reflejan una tendencia a la consideración individualizada y la recompensa contingente y no tanto a la dirección por excepción activa o pasiva (Avolio, Bass, \& Jung, 1999); sin embargo, aún es necesario que se incorporen más elementos del liderazgo transformacional, lo cual representa una oportunidad para la formación de líderes que sean fuente de inspiración, motivación y estimulación intelectual, es decir, líderes transformadores.
Finalmente, este estudio no solo permite identificar el estilo de liderazgo en Bucaramanga por medio de las percepciones de los subalternos, sino que también brinda información sobre cómo no es el liderazgo bumangués. Un hallazgo importante en este sentido es la baja elección de calificativos relacionados con la inspiración, pasión y predictibilidad por parte de los líderes. Estas características son deseables en el liderazgo del siglo XXI (Contreras, 2008). Ellas requieren ser potencializadas en estos líderes para tener un efecto transformador real sobre la calidad de vida de los trabajadores y el crecimiento y desarrollo de las organizaciones de la región. Gracias a este estudio es necesario indagar cuáles son las razones del bajo uso que hacen los subalternos del adjetivo 'distante' para describir a sus jefes. Esto no se espera en sociedades con alta distancia de poder, algo característico en Colombia y en el resto de Latinoamérica (The Hofstede Center, 2013), países en los que se acepta que el poder este distribuido de forma inequitativa.

\section{REFERENCIAS}

Avolio B., Bass B., \& Jung, D. (1999). Re-examining the components of transformational and transactional leadership using the Multifactor Leadership Question- 
naire. Journal of Occupational and Organizational Psychology, 72(4), 441-462.

Banco de la República - Biblioteca Virtual Luis Ángel Arango (2014). Sectoreseconómicos. Recuperado de http://www.banrepcultural.org/blaavirtual/ayudadetareas/economia/econo53.htm).

Barreiro de Souza, K., de Andrade Bastos, S., \& Salgueiro Perobelli, F. (2012). Análisis de la estructura productiva del sector servicios en países con diferentes niveles de desarrollo. Revista de la CEPAL, 108, 91-113.

Bass, B., \& Avolio, B. (1993). Transformational leadership and organizational culture. Public Administration Quarterly, 17,112-121.

Bass, B. (1999). Two decades of research and development in transformational leadership. European Journal of Work and Organizational Psychology, 8(1), 9-32.

Castro, A., \& Lupano, M. (2005). Diferencias individuales en las teorías implícitas del liderazgo y la cultura organizacional percibida. Boletín de Psicología, 85, 89-109.

Castro, A., \& Nader, M. (2004). Estilos de liderazgo, contexto y cultura organizacional. Un estudio comparativo en población civil y militar. Boletín de Psicología, 82, 45-65.
Contreras, F. (2008). Liderazgo: perspectivas de desarrollo e investigación. International Journal of Psychological Research, 1(2), 64-72.

Contreras, F., \& Barbosa, D. (2013). Del liderazgo transaccional al liderazgo transformacional: Implicaciones para el cambio organizacional. Revista Virtual Universidad Católica del Norte, 39, 152-164.

Contreras, F., Barbosa, D., Juárez, F., \& Uribe, A. (2010). Efectos del liderazgo y del clima organizacional sobre el riesgo psicosocial, como criterio de responsabilidad social, en empresas colombianas del sector salud. Revista Argentina de Clínica Psicológica, 19(2), 173-182.

Contreras, F., Barbosa, D., Juárez, F., Uribe, A., \& Mejía, C. (2009). Estilos de liderazgo, clima organizacional y riesgos psicosociales en entidades del sector salud, como criterios de responsabilidad social empresarial. Estudio comparativo en cuatro ciudades colombianas. RevistaActa ColombianadePsicología, 12(2), 13-26. Hofstede, G. (1991). Cultures and organizations: Software of the mind. Londres: McGraw-Hill.

Hofstede, G. (1994, January). Management scientists are human. $M a-$ nagement Science, 40(1), 4-13. 
Hofstede, G., Van Deusen, C. A. Mueller, C. B., \& Charles, T. A. (2002). What goals do business leaders pursue? A study in fifteen countries. Journal of International Business Studies, 33(4), 785 803

Javidan, M., Dorfman, P.W., Howell, J.P., \& Hanges, P.J. (2010). Leadership and cultural context. A Theoretical and empirical examination based on project GLOBE. EnN. Nohria\& R. Khurana(Eds.). Handbook of Leadership Theory and Practice (pp. 291-304). Boston: Harvard Business Press.

Juárez, F., Contreras F., \& Barbosa D. (2013). Propiedades psicométricas del test de adjetivos de Pitcher para la evaluación del liderazgo. International Journal of Psychological Research, 6(2), 109-116

Kogut, B., \& Singh, H. (1988). The effect of national culture on the choice of entry mode. Journal of International Business Studies, 19, 411-432.

Maroto-Sánchez, A. (2010). Growth and productivity in the service sector: The state of the art. Documentos de trabajo, $\mathrm{n}^{\circ}$ 7. Madrid: Universidad de Alcalá.

Martínez, Á., \& Lázaro, P. (2007). La ciencia de los servicios: un nuevo enfoque para la innovación en compañías de servicios. Universia Business Review, 15, 120-127.

Molero, F. (2002). Cultura y liderazgo. Una relación multifacética. Boletín de Psicología, 76, 53-75.

Pitcher, P. (1997a). The drama of leadership. Nueva York: John Wiley \& Sons.

Pitcher, P. (1997b). Management is a craft, not an art or science. $\mathrm{Ma}$ nagement Review, 86(8), 30-33.

Pitcher, P. (1999). Artists, craftsmen \& technocrats. Training \& Development, 53, 30-33.

Semenov, R. (2000). Cross-country differences in economic governance: Culture as a major explanatoryfactor(Tesisdedoctorado). Tilburg: Center for Economic Research, Tilburg University.

The Hofstede Center (2013). What about Colombia. Recuperado de http://geert-hofstede.com/colombia.html 